\title{
Cognitive diagnostic assessment based on knowledge structure
}

\author{
Sue-Fen Huang ${ }^{a}$ \\ Department of Digital Game and Animation Design, Taipei University of Marine Technology, Taiwan
}

\begin{abstract}
The purpose of this study is to provide an integrated method of fuzzy theory basis for individualized concept structure analysis. In order to insight the misconception of learning basic mathematics and progress teaching. This method integrates Fuzzy Logic Model of Perception (FLMP) and Interpretive Structural Modelling (ISM). The combined algorithm could analyze individualized concepts structure based on the comparisons with concept structure of expert. In this paper, some well-known knowledge structure assessment methods will be discussed. For item connection, Bart et al ordering theory and Takeya's item relational structure provided ordering coefficient to construct item relationships and hierarchies. For concepts or skills connection, Warfield's ISM and Lin et al Concept Advanced Interpretive Structural Modelling (CAISM) provided to construct graphic relationship among elements and display the individualized concept hierarchy structure by numeric and picture. Samples contain 427 which come from Min-Hwei Junior College. Subjects were analyzed by CAISM. It shows the traditional assessment is not the only criteria; it must be combined with other assessment tools. The result shows that CAISM gives meaningful learning and lacks of learners.
\end{abstract}

\section{Introduction}

Achievement assessment is a series of operations or examinations of learners' ability, knowledge, or learning outcome, consisting of questions for them to answer or activities for them to carry out. Achievement assessment perhaps is a most part of criteria for evaluating learners' learning performance, the people who has the same score will be classified into the same achievement group. However, there might exists distinct misconceptions from learners who have the same score, hence achievement assessment is no more a major criterion for evaluating learners' performance. Cognitive diagnostic assessment (CDA) is designed to obtain specific knowledge structures, processing skills and causes in terms of learners' understanding. Interpretation and explanation are required to providing feedback information for educator and learner which consisting of learners' lack of understanding or the primary cause of their performance [1][2].Cognition diagnosis can help represent knowledge structure [3] [4] [5]. It is a common viewpoint that human knowledge is stored in the form of structural relationship among concepts and their subordinate relationship is fuzzy, not crisp. There are some methodologies for concept structure analysis but little is known about methodologies of individualized concept structure [6] [7] [8] [9]. Therefore, the development for methodology of individualized concept structure is an important issue and it is essential for cognition

\footnotetext{
${ }^{a}$ Corresponding author : yih@mail.ntcu.edu.tw
} 
diagnosis and pedagogy [10]. In this study, the integrated method of individualized concept structure based on fuzzy logic model of perception (FLMP) and interpretive structural modelling (ISM) will be developed [11] [12] [13]. An example of empirical test data of linear algebra concept for students of learning deficiencies will also be analyzed and discussed. For the feasibility of remedial instruction based on the cognition diagnosis, clustering method is needed so that students within the same cluster own similar knowledge structures and students among different clusters have the most variance on knowledge structures [14].

\section{Literature review}

The utility of interpretive structural modelling (ISM) is to construct graphic relationship among elements within a complex system which was proposed by Warfield, J. N. [15] in 1982. For a subordinate matrix of a system, ISM will arrange elements in the form of hierarchical structure. Lin, Y. H., Hung, W. L., \& Huang, K. J. (2006) [16] extended Warfield's interpretive structural modelling and proposed concept advanced interpretive structural modelling. The object of concept advanced interpretive structural modelling (CAISM) is to provide individualized hierarchy structure of knowledge of examinees based on response patterns of tests. CAISM incorporates principles of concept vector matching and $\alpha$-cut of fuzzy set theory into ISM to display the individualized concept hierarchy structure by numeric and picture.

\subsection{Concept advanced interpretive structural modelling}

The definition of data structures are described as follows.

$X=\left[x_{n m}\right]_{N \times M}$ is the response matrix of all examinees on the test. There exist $M$ items in a test and $N$ examinees. We know that $x_{n m}=1$ when examinee $n$ gives correct answer on item $m$; otherwise $x_{n m}=0$ means examinee $n$ gives wrong answer on item $m . Y=\left[y_{m a}\right]_{M \times A}$ is the item-concept matrix, which containing $A$ concepts. If item $m$ measures concept $a$, then $y_{m a}$ is denoted by 1 ; otherwise $y_{m a}$ is denoted by $0 . Z=\left[z_{i a}\right]_{I \times A}$ is the ideal concept matrix, where $z_{i}=\left(z_{i 1}, z_{i 2}, \ldots, z_{i A}\right), i=1,2, \ldots, I, I=$ $2^{A}$. if ideal concept vector $z_{i}$ contains concept a then $z_{i a}=1$; otherwise $z_{i a}=0 . R=\left[r_{i m}\right]_{I \times M}$ is the ideal response matrix and $r_{i}=\left(r_{i 1}, r_{i 2}, \ldots, r_{i M}\right)$ is the ideal response vector. Ideal response vector is the response pattern on all items based on corresponding ideal concept vector $z_{i}$. It is $r_{i m}=1$ if ideal concept vector $z_{i}$ could provide correct answer on item $m$; otherwise $r_{i m}=0 \cdot S C=\left[s c_{n i}\right]_{N \times I}$ is the standardized closeness matrix which measures the closeness between response vector $x_{n}=\left(x_{n 1}, x_{n 2}, \ldots, x_{n M}\right)$ and ideal response vector $r_{i}=\left(r_{i 1}, r_{i 2}, \ldots, r_{i M}\right)$. Greater $s c_{n i}$ means more similarity between $x_{n}$ and $r_{i}$. The algorithm of CAISM is described as follows:

The ideal response matrix is defined

$$
\begin{gathered}
r_{i m}=\left\{\begin{array}{c}
1, z_{i a} \cdot y_{m a}=y_{m a} \\
0 \quad \text { otherwise }
\end{array}, \forall a=1,2, \ldots, A\right. \\
c_{n i}=\frac{\sum_{m=1}^{M} x_{n m} \circ r_{i m}}{M}
\end{gathered}
$$

where 


$$
x_{n m} \circ r_{i m}= \begin{cases}1 & , x_{n m}=r_{i m} \\ 0 & , x_{n m} \neq r_{i m}\end{cases}
$$

$c_{n i}$ is the closeness between the $x_{n}$ and $r_{i}$, then the standardized closeness $S c_{n i}$

(a) It is crisp recognition if $K(K \geq 1)$ different $c_{n i}$ values satisfy $c_{n i}=1$, and

$$
s c_{n i}= \begin{cases}1 & , \forall c_{n i}=1 \\ 0 & , \text { otherwise }\end{cases}
$$

(b) It is fuzzy recognition if $c_{n i} \neq 1$

$$
s c_{n i}=\frac{c_{n i}}{\sum_{i=1}^{I} c_{n i}}, \quad \forall i=1,2, \ldots, I
$$

\subsection{ISM approach on concept structure analysis}

J. N. Warfield provided the foundation of ISM [17]. The integrated algorithms consist of three steps of algorithms, AMC, ASC and AFISM.

\section{Knowledge structure analysis}

The test includes 19 items with 427 task-takers of College students. The data set comes from the MinHwei Junior College. used in the empirical study with learning Abstract Algebra. There are 7 concept attributes within each item and they are depicted in Table1.

Table 1. The Details of the Used Datasets.

\begin{tabular}{|c|l|}
\hline Classes & Characters about Concepts \\
\hline 1 & Number \\
\hline 2 & Coordinates \\
\hline 3 & Geometry \\
\hline 4 & Function \\
\hline 5 & Distance \\
\hline 6 & Statistics \\
\hline 7 & Equation \\
\hline
\end{tabular}

As shown from Figure 1, To student 139, All concepts are equal. All concepts are so easy to the student. The index of diffcult is closed to zero. The concept of structure is very like expert.

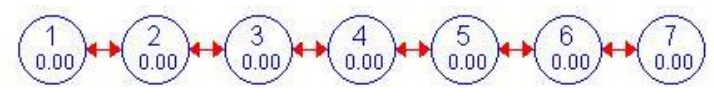

Figure 1. Knowledge Structure of Student 139.

As shown from Figure 2, To student 378, mastery of concept 5 and 6 are 0.50 . Concept 5 and 6 are the basis for concept 2,7. Concept 1 is difficult for student 378. It is also clearly understood that concept structures of these two students vary a lot. 


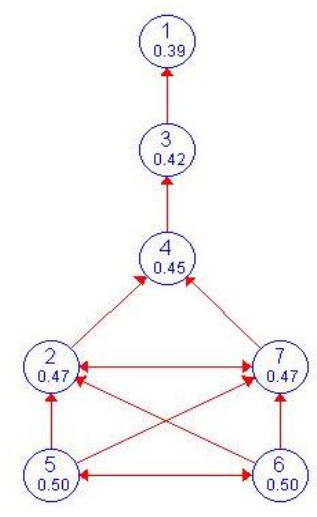

Figure 2. Knowledge Structure of Student 378.

\section{Conclusion}

Each cluster of data can easily describe features of knowledge structures. Manage the knowledge structures of Mathematics Concepts to construct the model of features in the pattern recognition completely. To sum up, this integrated algorithm could improve the assessment methodology of cognition diagnosis and manage the knowledge structures of Mathematics Concepts easily. It shows the traditional assessment is not the only criteria; it must be combined with other assessment tools. The result shows that CAISM gives meaningful learning and lacks of learners.

\section{References}

1. J.P. Leighton and M.J. Gierl, Cambridge University Press, 3-18 (2007)

2. S.P. Norris, J.S. Macnab and L.M. Phillips, Cognitive diagnostic assessment for education: Theory and practices, Cambridge University Press, 61-84 (2007)

3. M. Smithson and J. Verkuilen, Fuzzy Set Theory: Applications in the Social Sciences, Sage Publications (2006)

4. R. Coppi, P. Giordani and P. D’Urso, Psychometrika, 71, 733-761 (2006)

5. T. Sato, The S-P Chart and The Caution Index, NEC Educational Information Bulletin 80-1, C\&C Systems Research Laboratories. Nippon Electic Co., Ltd,. Tokyo, Japan (1980)

6. J.P. Doignon and J.C. Falmagne, Knowledge Space. Springer-Verlag (1999)

7. K. VanLehn, Journal of the Learning Sciences, 8, 71 (1999)

8. R.W. Schvaneveldt, Pathfinder Associative Networks. Ablex (1991)

9. W.P. Jr. Fisher, Rasch Measurement Transactions, 9, 442 (1995)

10. R.J. Mislevy and N. Verhelst, Psychometrika, 55, 195 (1990)

11. J.N. Warfield, Societal Systems Planning. Policy and Complexity, Wiley (1976)

12. J.N. Warfield, Interpretive Structural Modeling (ISM). In S. A. Olsen (Eds.), Group Planning \& Problem Solving Methods in Engineering, 155-201, Wiley (1982)

13. L.A. Zadeh, Information and Control, 8, 338 (1965)

14. Y.H. Lin, M.W. Bart and K.J. Huang, Generalized Polytomous Ordering Theory. [manual and software], National Taichung University, Taiwan (2006)

15. J.N. Warfield, Interpretive structural modeling. In S. A. Olsen (Ed.), Group Planning and Problem-solving Methods in Engineering, 155-201, New York: Wiley (1982)

16. Y.H. Lin, W.L. Hung and K.J. Huang, CAISM software [Manual and Software for CAISM]. Taiwan, Taichung City: National Taichung University,(2006)

17. J.N. Warfield, Crossing Theory and Hierarchy Mapping, 7, 505 (1977) 